\title{
A Liouville theorem for Lévy generators
}

\author{
Franziska Kühn ${ }^{1}[$
}

Received: 7 February 2020 / Accepted: 28 November 2020 / Published online: 8 December 2020

(c) The Author(s) 2020

\section{Abstract}

Under mild assumptions, we establish a Liouville theorem for the "Laplace" equation $A u=0$ associated with the infinitesimal generator $A$ of a Lévy process: If $u$ is a weak solution to $A u=0$ which is at most of (suitable) polynomial growth, then $u$ is a polynomial. As a by-product, we obtain new regularity estimates for semigroups associated with Lévy processes.

Keywords Liouville theorem $\cdot$ Pseudo-differential operator $\cdot$ Lévy process

Mathematics Subject Classification Primary 60G51 - 35B53; Secondary 31C05 . 35R09 $\cdot 60 \mathrm{~J} 35$

\section{Introduction}

The classical Liouville theorem states that any bounded solution $u: \mathbb{R}^{d} \rightarrow \mathbb{R}$ to the Laplace equation $\Delta u=0$ is constant. There is an extension for unbounded functions: If $\Delta u=0$ and $u$ is at most of polynomial growth, say, $|u(x)| \leq C\left(1+|x|^{k}\right)$ for some constants $C>0$ and $k \in \mathbb{N}_{0}$, then $u$ is a polynomial of degree at most $k$. In this paper, we extend this result to a wide class of integro-differential operators. More precisely, we establish a Liouville theorem for equations $A u=0$ where $A$ is of the form

$$
\begin{aligned}
A f(x)= & b \cdot \nabla f(x)+\frac{1}{2} \operatorname{tr}\left(Q \cdot \nabla^{2} f(x)\right) \\
& +\int_{y \neq 0}\left(f(x+y)-f(x)-y \cdot \nabla f(x) \mathbb{1}_{(0,1)}(|y|)\right) v(d y), f \in C_{c}^{\infty}\left(\mathbb{R}^{d}\right),
\end{aligned}
$$

Franziska Kühn

franziska.kuehn1@tu-dresden.de

1 TU Dresden, Fakultät Mathematik, Institut für Mathematische Stochastik, 01062 Dresden, Germany 
for some $b \in \mathbb{R}^{d}$, a positive semi-definite matrix $Q \in \mathbb{R}^{d \times d}$ and a measure $v$ on $\left(\mathbb{R}^{d} \backslash\{0\}, \mathcal{B}\left(\mathbb{R}^{d} \backslash\{0\}\right)\right)$ satisfying $\int_{y \neq 0} \min \left\{1,|y|^{2}\right\} \nu(d y)<\infty$. Equivalently, $A$ can be written as a pseudo-differential operator,

$$
A f(x)=-\psi(D) f(x):=-\int_{\mathbb{R}^{d}} \psi(\xi) e^{i x \cdot \xi} \hat{f}(\xi) d \xi, \quad f \in C_{c}^{\infty}\left(\mathbb{R}^{d}\right), x \in \mathbb{R}^{d},
$$

where $\hat{f}(\xi)=(2 \pi)^{-d} \int_{\mathbb{R}^{d}} f(x) e^{-i x \cdot \xi} d x$ denotes the Fourier transform of $f$ and the symbol $\psi$ is a continuous negative definite function with Lévy-Khintchine representation

$$
\psi(\xi)=i b \cdot \xi+\frac{1}{2} \xi \cdot Q \xi+\int_{y \neq 0}\left(1-e^{i y \cdot \xi}+i y \cdot \xi \mathbb{1}_{(0,1)}(|y|)\right) v(d y), \quad \xi \in \mathbb{R}^{d} .
$$

Since $A$ is the infinitesimal generator of a Lévy process, see below, we also call $A$ a Lévy generator. The family of Lévy generators includes many interesting and important operators, e.g. the Laplacian $\Delta$, the fractional Laplacian $-(-\Delta)^{\alpha / 2}, \alpha \in(0,2)$, and the free relativistic Hamiltonian $m-\sqrt{-\Delta+m^{2}}, m>0$. If $A$ is a local operator, i.e. $v=0$, then the Liouville theorem is classical, and so the focus is on the nonlocal case $v \neq 0$. For Lévy generators with a sufficiently smooth symbol, there is a Liouville theorem by Fall and Weth [5]; the required regularity of $\psi$ increases with the dimension $d \in \mathbb{N}$. Ros-Oton and Serra [19] established a general Liouville theorem for symmetric stable operators,

$$
A f(x)=\int_{\mathbb{S}^{d-1}} \int_{(0, \infty)}(f(x+\theta r)+f(x-\theta r)-2 f(x)) \frac{d r}{r^{d+\alpha}} \mu(d \theta),
$$

where $\alpha \in(0,2)$ and $\mu$ is a non-negative finite measure on the unit sphere $\mathbb{S}^{d-1}$ satisfying an ellipticity condition. Liang and Wang [16, Corollary 1.3] established a Liouville theorem under the assumption that $v$ is bounded below and above by a stable measure. The recent papers $[1,11]$ give necessary and sufficient conditions for the Liouville property, i.e. conditions under which the implication

$$
u \in L^{\infty}\left(\mathbb{R}^{d}\right), A u=0 \text { weakly } \Longrightarrow u \text { is constant }
$$

holds. Choquet and Deny [4] characterized the bounded solutions $u$ to convolution equations of the form $u=u * \mu$; these equations play a central role in the study of the "Laplace" equation $A u=0$, see Lemma 1. Since the Liouville theorem is an assertion on the smoothness of harmonic functions, there is a close connection between the Liouville theorem and Schauder estimates; see [13,19] and the references therein for recent results. We would like to mention that there are also Liouville theorems in the half-space, see e.g. [3,19], and Liouville theorems for certain Lévy-type operators, see e.g. $[2,17,18,23]$.

In this paper, we use a probabilistic approach, inspired by [19], to prove a Liouville theorem for a wide class of Lévy generators. Before stating the result, let us briefly recall some material from probability theory. It is well known, cf. $[8,9,20]$, that there is a one-to-one correspondence between continuous negative definite functions and Lévy 
processes, i.e. stochastic processes with càdlàg (right-continuous with finite left-hand limits) sample paths and stationary and independent increments. Given a continuous negative definite function $\psi: \mathbb{R}^{d} \rightarrow \mathbb{C}$, there exists a Lévy process $\left(X_{t}\right)_{t \geq 0}$ with semigroup $P_{t} f(x):=\mathbb{E} f\left(x+X_{t}\right)$ satisfying

$$
-\psi(D) f(x)=\lim _{t \rightarrow 0} \frac{P_{t} f(x)-f(x)}{t}, \quad f \in C_{c}^{\infty}\left(\mathbb{R}^{d}\right), x \in \mathbb{R}^{d},
$$

which means that $A=-\psi(D)$ is the infinitesimal generator of $\left(X_{t}\right)_{t \geq 0}$. The Lévy process $\left(X_{t}\right)_{t \geq 0}$ is uniquely determined by $\psi$, the so-called characteristic exponent of $\left(X_{t}\right)_{t \geq 0}$, and by the associated Lévy triplet $(b, Q, v)$. The following theorem is our main result.

Theorem 1 Let $\left(X_{t}\right)_{t \geq 0}$ be a Lévy process with Lévy triplet $(b, Q, v)$ and characteristic exponent $\psi$, and denote by $A f=-\psi(D) f$ the associated Lévy generator. Assume that

(C1) $X_{t}$ has for each $t>0$ a density $p_{t} \in C_{b}^{1}\left(\mathbb{R}^{d}\right)$ with respect to Lebesgue measure,

(C2) there exists some $\beta>0$ such that $\int_{|y| \geq 1}|y|^{\beta} \nu(d y)<\infty$.

If $u: \mathbb{R}^{d} \rightarrow \mathbb{R}$ is a weak solution to

$$
A u=0 \text { in } \mathbb{R}^{d}
$$

satisfying $|u(x)| \leq M\left(1+|x|^{\gamma}\right), x \in \mathbb{R}^{d}$, for some $M>0$ and $\gamma \in[0, \beta)$, then $u$ is a polynomial of degree at most $\lfloor\gamma\rfloor$. In particular, A has the Liouville property (4).

Remark 1 (i). Weak solutions to $A u=0$ are only determined up to a Lebesgue null set, cf. Sect. 2. When we write " $u$ is a polynomial", this means that $u$ has a representative which is a polynomial, i.e. there is a polynomial $\tilde{u}$ such that $u=\tilde{u}$ Lebesgue almost everywhere.

(ii). A sufficient condition for (C1) is the Hartman-Wintner condition,

$$
\lim _{|\xi| \rightarrow \infty} \frac{\operatorname{Re} \psi(\xi)}{\log (|\xi|)}=\infty
$$

see [10] for a thorough discussion.

(iii). Condition (C2) is equivalent to assuming that $\mathbb{E}\left(\left|X_{t}\right|^{\beta}\right)=\int_{\mathbb{R}^{d}}|x|^{\beta} p_{t}(x) d x$ is finite for some (all) $t>0$, cf. [20]. Consequently, (C2) implies, in particular, that $P_{t} u(x)=\mathbb{E} u\left(x+X_{t}\right)$ is well defined for any measurable function $u$ satisfying the growth condition $|u(x)| \leq M\left(1+|x|^{\beta}\right)$.

(iv). In Theorem 1 we impose an integral condition on $v$ and a pointwise growth condition on $u$. One can also think of imposing a pointwise growth condition on (the density of) $v$ and an integral condition on $u$, see [5] for a result in this direction.

(v). The conditions (C1) and (C2) are quite mild assumptions, which hold for a large class of pseudo-differential operators. The recent paper [11] does, however, indicate that our conditions are not sharp; it is shown that $A=-\psi(D)$ has the Liouville property (4) iff $\{\psi=0\}=\{0\}$. By the Riemann-Lebesgue lemma, (C1) implies $\{\psi=0\}=\{0\}$ but the converse is not true. 
Example 1 (i). If $\left(X_{t}\right)_{t \geq 0}$ is a Brownian motion, then (C1) is trivial and (C2) holds for all $\beta>0$. The associated Lévy generator is the Laplacian and, consequently, we recover the classical Liouville theorem for the Laplacian.

(ii). Consider an $\alpha$-stable operator $A$ of the form (3) for a finite spectral measure $\mu$ on $\mathbb{S}^{d-1}$ which is non-degenerate, in the sense that its support is not contained in any proper linear subspace of $\mathbb{R}^{d}$. Then the assumptions of Theorem 1 are satisfied for $\beta<\alpha$, and we recover the Liouville theorem for stable operators in [19, Theorem 2.1].

(iii). Let $\alpha_{1}, \ldots, \alpha_{d} \in(0,2)$. The anisotropic operator $A u=-\sum_{k=1}^{d}\left(-\partial_{x_{k}}^{2} u\right)^{\alpha_{k} / 2}$ appears as Lévy generator of the Lévy process with characteristic exponent $\psi(\xi)=$ $\sum_{k=1}^{d}\left|\xi_{k}\right|^{\alpha_{k}}, \xi=\left(\xi_{1}, \ldots, \xi_{d}\right) \in \mathbb{R}^{d}$. Since $\psi$ satisfies the Hartman-Wintner condition (5), it follows that (C1) holds; moreover, the associated Lévy measure $v$ satisfies the moment condition (C2) for $\beta<\min _{k} \alpha_{k}$.

(iv). If the Lévy measure $v$ of a Lévy generator satisfies

$$
v(B) \geq \int_{|y|<1} \frac{1}{|y|^{d+\alpha}} \mathbb{1}_{B}(y) d y, \quad B \in \mathcal{B}\left(\mathbb{R}^{d} \backslash\{0\}\right),
$$

for some $\alpha>0$, then (5) holds and, in consequence, (C1) is satisfied. A sufficient condition for (C2) is, for instance, that $\nu(d y)=g(y) d y$ with $g(y) \leq C|y|^{-d-\gamma}$, $|y| \gg 1$, for some constants $C>0$ and $\gamma>\beta$.

Let us sketch the idea of the proof of Theorem 1. First, we show under mild assumptions that every weak solution to the equation $A u=0$ gives rise to a (continuous) solution to the convolution equation $P_{t} u=u$. The intuition behind this result comes from Dynkin's formula: If $A u=0$ and $u$ is, say, twice differentiable and bounded, then Dynkin's formula, cf. [8, Lemma 4.1.14], shows

$$
P_{t} u-u=\int_{0}^{t} P_{s} A u d s=0 \quad \text { for all } t \geq 0 .
$$

Secondly, we use that the convolution operator $P_{t}$ has smoothing properties, i.e. $P_{t} u$ has a higher regularity than $u$. If $u$ is a solution to $A u=0$, and hence to $P_{t} u=u$, then these regularizing properties of $P_{t}$ allow us to establish suitable Hölder estimates for $u$ which lead, by iteration, to the conclusion that $u$ is smooth; thus a polynomial.

The remaining article is structured as follows. In Sect. 2 we introduce the notion of weak solutions and study the connection between the "Laplace" equation $A u=0$ and the convolution equation $P_{t} u=u$. In Sect. 3 we establish regularity estimates for the semigroup $\left(P_{t}\right)_{t \geq 0}$, which are of independent interest. The Liouville theorem is proved in Sect. 4.

\section{Weak solutions}

Let $A=-\psi(D)$ be a pseudo-differential operator with continuous negative definite symbol $\psi: \mathbb{R}^{d} \rightarrow \mathbb{C}$, cf. (2). Since $\overline{\psi(\xi)}=\psi(-\xi)$ for all $\xi \in \mathbb{R}^{d}$, an application of 
Plancherel's theorem shows that the pseudo-differential operator $A^{*} f:=-\bar{\psi}(D) f$ is the adjoint of $A$ in $L^{2}(d x)$. Indeed, if $\varphi, f \in C_{c}^{\infty}\left(\mathbb{R}^{d}\right)$, then

$$
\langle A f, \varphi\rangle_{L^{2}}=\langle\widehat{A f}, \check{\varphi}\rangle_{L^{2}}=\langle-\psi \hat{f}, \check{\varphi}\rangle_{L^{2}}=\langle\hat{f},-\psi \check{\varphi}\rangle_{L^{2}}=\left\langle\hat{f}, \bar{A}^{*} \varphi\right\rangle_{L^{2}}=\left\langle f, A^{*} \varphi\right\rangle_{L^{2}}
$$

where $\check{\varphi}$ denotes the inverse Fourier transform of $\varphi$.

Definition 1 Let $A$ be a pseudo-differential operator with continuous negative definite symbol $\psi: \mathbb{R}^{d} \rightarrow \mathbb{C}$. Let $U \subseteq \mathbb{R}^{d}$ be open and $f \in L_{\text {loc }}^{1}(U)$. A measurable function $u: \mathbb{R}^{d} \rightarrow \mathbb{R}$ is a weak solution to

$$
A u=f \quad \text { in } U
$$

if

$$
\forall \varphi \in C_{c}^{\infty}(U): \int_{\mathbb{R}^{d}} u(x) A^{*} \varphi(x) d x=\int_{U} f(x) \varphi(x) d x .
$$

In (6) we implicitly assume that the integrals exist. For the integral on the right-hand side, the existence is evident from $\varphi \in C_{c}^{\infty}(U)$ and $f \in L_{\text {loc }}^{1}(U)$. The other integral is harder to deal with because $A^{*}$ is a non-local operator, i.e. decay properties of $\varphi$ (e.g. compactness of the support) do not carry over to $A^{*} \varphi$. Our first result in this section shows that the decay of $A^{*} \varphi$ is closely linked to the existence of fractional moments $\int_{|y| \geq 1}|y|^{\beta} v(d y)$ of the Lévy measure $v$, associated with $\psi$ via (2); see [5, Lemma 2.1] for a related result.

Proposition 1 Let $\psi: \mathbb{R}^{d} \rightarrow \mathbb{C}$ be a continuous negative definite function with triplet $(b, Q, v)$. If $\beta>0$ is such that $\int_{|y| \geq 1}|y|^{\beta} v(d y)<\infty$, then the pseudo-differential operator $A=-\psi(D)$ satisfies

$$
\int_{\mathbb{R}^{d}}\left(1+|x|^{\beta}\right)|A \varphi(x)| d x<\infty \quad \text { for all } \varphi \in C_{c}^{\infty}\left(\mathbb{R}^{d}\right) .
$$

More precisely, there is for all $R>0$ a constant $C>0$ such that every $\varphi \in C_{c}^{\infty}\left(\mathbb{R}^{d}\right)$ with $\operatorname{supp} \varphi \subset B(0, R)$ satisfies

$$
\begin{aligned}
& \int_{\mathbb{R}^{d}}\left(1+|x|^{\beta}\right)|A \varphi(x)| d x \\
& \quad \leq C\|\varphi\|_{C_{b}^{2}\left(\mathbb{R}^{d}\right)}\left(|b|+|Q|+\int_{|y| \leq 1}|y|^{2} v(d y)+\int_{|y|>1}|y|^{\beta} v(d y)\right) .
\end{aligned}
$$

Let us mention that $\int_{|y| \geq 1}|y|^{\beta} \nu(d y)<\infty$ is actually equivalent to (7). Here, we need (and prove) only sufficiency for (7); for the converse implication see [6, Theorem 4.1]. Proposition 1 gives a sufficient condition such the integral on the left-hand side of (6) exists: Since the adjoint $A^{*}$ is a pseudo-differential operator with symbol $\bar{\psi}$ and triplet $(-b, Q, v(-\cdot))$, Proposition 1 shows that $\int_{\mathbb{R}^{d}}|u(x)|\left|A^{*} \varphi(x)\right| d x$ is finite for 
every measurable function $u$ satisfying $|u(x)| \leq M\left(1+|x|^{\beta}\right)$ for some $\beta \geq 0$ with $\int_{|y| \geq 1}|y|^{\beta} v(d y)<\infty$.

Proof of Proposition 1 Since the assertion is obvious for the local part of $A$, we may assume without loss of generality that $b=0$ and $Q=0$. Fix $\varphi \in C_{c}^{\infty}\left(\mathbb{R}^{d}\right)$ with $\operatorname{supp} \varphi \subset B(0, R)$. For $x \in \mathbb{R}^{d}$ with $|x| \geq 2 R$, we have

$$
\begin{aligned}
|x|^{\beta}|A \varphi(x)| & \leq|x|^{\beta} \int_{|y+x|<R}|\varphi(x+y)| v(d y) \\
& \leq \int_{|y| \geq|x|-R}|\varphi(x+y)| \frac{|x|^{\beta}}{(|x|-R)^{\beta}}|y|^{\beta} v(d y) \\
& \leq C \int_{|y| \geq R}|\varphi(x+y)||y|^{\beta} v(d y)
\end{aligned}
$$

for some constant $C=C(R)$. Integrating with respect to $x$, we find by Tonelli's theorem that

$$
\int_{|x| \geq 2 R}|x|^{\beta}|A \varphi(x)| d x \leq C\|\varphi\|_{\infty}(2 R)^{d} \int_{|y| \geq R}|y|^{\beta} v(d y) .
$$

On the other hand, it is immediate from Taylor's formula that

$$
\|A \varphi\|_{\infty} \leq 2\|\varphi\|_{C_{b}^{2}(\mathbb{R})} \int_{y \neq 0} \min \left\{1,|y|^{2}\right\} \nu(d y),
$$

and this yields the required estimate for $\int_{|x|<2 R}\left(1+|x|^{\beta}\right)|A \varphi(x)| d x$.

Next we establish a connection between the "Laplace" equation $A u=0$ and the convolution equation $P_{t} u=u$.

Lemma 1 Let $\left(X_{t}\right)_{t>0}$ be a Lévy process with Lévy triplet $(b, Q, v)$, infinitesimal generator $(A, \mathcal{D}(A))$ and semigroup $\left(P_{t}\right)_{t \geq 0}$. Assume that $X_{t}$ has for $t>0 a$ density $p_{t} \in C_{b}\left(\mathbb{R}^{d}\right)$ with respect to Lebesgue measure, and let $\beta \geq 0$ be such that $\int_{|y| \geq 1}|y|^{\beta} v(d y)<\infty$. If $u: \mathbb{R}^{d} \rightarrow \mathbb{R}$ is a measurable function with $|u(x)| \leq M\left(1+|x|^{\beta}\right), x \in \mathbb{R}^{d}$, solving

$$
A u=0 \text { weakly in } \mathbb{R}^{d},
$$

then there exists $\tilde{u} \in C\left(\mathbb{R}^{d}\right)$ such that $u=\bar{u}$ Lebesgue almost everywhere and $\tilde{u}=P_{t} \tilde{u}$ for all $t>0$. 
Note that the exceptional null set $\left\{\tilde{u} \neq P_{t} \tilde{u}\right\}$ does, in general, depend on $t$; for the application which we have in mind, that is, for the proof of Liouville's theorem, this is not a problem since we will use the result only for $t=1$.

Proof Take $\varphi \in C_{c}^{\infty}\left(\mathbb{R}^{d}\right)$ such that $\varphi \geq 0$ and $\int_{\mathbb{R}^{d}} \varphi(x) d x=1$. Set $\varphi_{\varepsilon}(x):=$ $\varepsilon^{-d} \varphi(x / \varepsilon)$ and

$$
u_{\varepsilon}(x):=\left(u * \varphi_{\varepsilon}\right)(x):=\int_{\mathbb{R}^{d}} u(x-y) \varphi_{\varepsilon}(y) d y, \quad x \in \mathbb{R}^{d},
$$

for $\varepsilon>0$. Using

$$
(a+b)^{\beta} \leq c_{\beta}\left(a^{\beta}+b^{\beta}\right), \quad a, b \geq 0,
$$

it follows that

$$
\begin{aligned}
\left|u_{\varepsilon}(x)\right| & \leq M \int_{\mathbb{R}^{d}}\left(1+|x-y|^{\beta}\right) \mid \varphi_{\varepsilon}(y) d y \\
& \leq M c_{\beta}\left(1+|x|^{\beta}\right)\left(\int_{\mathbb{R}^{d}}\left|\varphi_{\varepsilon}(y)\right| d y+\int_{\mathbb{R}^{d}}|y|^{\beta}\left|\varphi_{\varepsilon}(y)\right| d y\right) \\
& \leq C_{1}\left(1+|x|^{\beta}\right)
\end{aligned}
$$

for a constant $C_{1}>0$ not depending on $\varepsilon, x$ and $u$. As $\int_{|y| \geq 1}|y|^{\beta} v(d y)<\infty$, the Lévy process has fractional moments of order $\beta$, i.e. $\mathbb{E}\left(\left|X_{t}\right|^{\beta}\right)=\int|y|^{\beta} p_{t}(y) d y$ is finite, see e.g. [20, Theorem 25.3] or [12, Theorem 4.1], and so $P_{t} u$ and $P_{t} u_{\varepsilon}$ are well-defined. We have

$$
\begin{aligned}
\left|P_{t} u(x)-P_{t} u_{\varepsilon}(x)\right| \leq & \left\|p_{t}\right\|_{\infty} \int_{|y| \leq R}\left|u(y)-u_{\varepsilon}(y)\right| d y \\
& +2 M \int_{|y|>R}\left(1+|y|^{\beta}\right) p_{t}(y-x) d y .
\end{aligned}
$$

For fixed $x \in \mathbb{R}^{d}$, it follows from the dominated convergence theorem that the second term on the right-hand side is less than, say, $\varrho>0$, for $R$ large enough. Since $u_{\varepsilon} \rightarrow u$ in $L_{\mathrm{loc}}^{1}(d x)$, the first term is less than $\varrho$ for small $\varepsilon>0$. Hence, $P_{t} u_{\varepsilon}(x) \rightarrow P_{t} u(x)$ as $\varepsilon \rightarrow 0$ for each $x \in \mathbb{R}^{d}$. Next we show that

$$
P_{t} u_{\varepsilon}(x)=u_{\varepsilon}(x) \quad \text { for all } t>0, x \in \mathbb{R}^{d}, \varepsilon>0 \text {. }
$$

By the definition of $P_{t} u$ and $u_{\varepsilon}$,we have

$$
P_{t} u_{\varepsilon}(x)=\int_{\mathbb{R}^{d}}\left(\int_{\mathbb{R}^{d}} u(z) \varphi_{\varepsilon}(y-z) d z\right) p_{t}(y-x) d y .
$$

Because of the growth estimate in (9), we may apply Fubini's theorem:

$$
P_{t} u_{\varepsilon}(x)=\int_{\mathbb{R}^{d}}\left(\int_{\mathbb{R}^{d}} \varphi_{\varepsilon}(y-z) p_{t}(y-x) d y\right) u(z) d z
$$




$$
=u_{\varepsilon}(x)+\int_{\mathbb{R}^{d}} u(z)\left(\mathbb{E} \varphi_{\varepsilon}\left(x-z+X_{t}\right)-\varphi_{\varepsilon}(x-z)\right) d z=: u_{\varepsilon}(x)+\Delta .
$$

It remains to show that $\Delta=0$. As $\varphi_{\varepsilon} \in C_{c}^{\infty}\left(\mathbb{R}^{d}\right)$, an application of Dynkin's formula gives

$$
\Delta=\int_{\mathbb{R}^{d}} u(z) \int_{0}^{t} \mathbb{E}\left(\left(A \varphi_{\varepsilon}\right)\left(x-z+X_{s}\right)\right) d s d z
$$

Applying Lemma 1, using the growth condition on $u$ and the fact that $\int_{0}^{t} \mathbb{E}\left(\left|X_{s}\right|^{\beta}\right) d s$ is finite, cf. [20, Theorem 25.18] or [12, Theorem 4.1], we find that

$$
\mathbb{E}\left(\int_{0}^{t} \int_{\mathbb{R}^{d}}\left|u\left(z+X_{s}\right)\right|\left|\left(A \varphi_{\varepsilon}\right)(x-z)\right| d z d s\right)<\infty
$$

and therefore we may apply once more Fubini's theorem:

$$
\Delta=\mathbb{E}\left(\int_{0}^{t} \int_{\mathbb{R}^{d}}\left(A \varphi_{\varepsilon}\right)\left(x-z+X_{s}\right) u(z) d z d s\right) .
$$

From

$$
(A \phi)(y-z)=(A \phi(\bullet+y))(-z) \text { and }(A \phi)(-z),=\left(A^{*} \phi(-\bullet)\right)(z) \text {. }
$$

we conclude that

$$
\Delta=\mathbb{E}\left(\int_{0}^{t} \int_{\mathbb{R}^{d}}\left(A^{*} \varphi_{\varepsilon}\left(x+X_{s}-\bullet\right)\right)(z) u(z) d z d s\right) .
$$

Since $z \mapsto \varphi_{\varepsilon}\left(x+X_{s}(\omega)-z\right) \in C_{c}^{\infty}\left(\mathbb{R}^{d}\right)$ for each fixed $\omega \in \Omega, s \in[0, t]$ and $x \in \mathbb{R}^{d}$, it follows from $A u=0$ weakly that the inner integral on the right-hand side is zero, and so $\Delta=0$. This finishes the proof of (10). As $u_{\varepsilon} \rightarrow u$ in $L_{1}^{\text {loc }}$, there exists a subsequence converging Lebesgue almost everywhere. Letting $\varepsilon \rightarrow 0$ in (10) along this subsequence, we get $P_{t} u=u$ Lebesgue almost everywhere. If we set $\tilde{u}:=P_{1} u$, then $u=P_{1} u=\tilde{u}$ Lebesgue almost everywhere and

$$
\tilde{u}=u=P_{t} u=P_{t} \tilde{u} \quad \text { a.e. }
$$

where the latter equality follows from the fact that $P_{t}$ does not see Lebesgue null sets since $X_{t}$ has a density with respect to Lebesgue measure. Finally, we note that $\tilde{u} \in C\left(\mathbb{R}^{d}\right)$. Indeed, given $\varepsilon>0$ and $r>0$, there is some $R>r$ such that

$$
\sup _{x \in B(0, r)} \int_{|y| \geq R}\left(1+|y|^{\beta}\right) p_{1}(y-x) d y=\sup _{x \in B(0, r)} \int_{|y| \geq R}\left(1+|y+x|^{\beta}\right) p_{1}(y) d y \leq \epsilon .
$$


Hence, for all $x, z \in B(0, r)$

$$
\begin{aligned}
& |\tilde{u}(x)-\tilde{u}(z)| \leq \int_{|y| \leq R}|u(y)|\left|p_{1}(y-x)-p_{1}(y-z)\right| d y \\
& +\int_{|y| \geq R}|u(y)|\left|p_{1}(y-x)-p_{1}(y-z)\right| d y \\
& \leq M\left(1+R^{\beta}\right) R^{d} \sup _{\substack{|u-v| \leq|x-z| \\
u, v \in B(0,2 R)}}\left|p_{1}(u)-p_{1}(v)\right|+2 M \epsilon \\
& \stackrel{|x-z| \rightarrow 0}{\longrightarrow} 2 M \epsilon \stackrel{\varepsilon \rightarrow 0}{\longrightarrow} 0,
\end{aligned}
$$

i.e. $\tilde{u}$ is continuous. Since $\tilde{u}$ and $P_{t} \tilde{u}$ are continuous, it follows from $\tilde{u}=P_{t} \tilde{u}$ Lebesgue almost everywhere that $\tilde{u}(x)=P_{t} \tilde{u}(x)$ for all $x \in \mathbb{R}^{d}$.

\section{Regularity estimates for semigroups associated with Lévy processes}

Let $\left(X_{t}\right)_{t \geq 0}$ be a Lévy process with transition density $p_{t}, t>0$, and semigroup

$$
P_{t} u(x):=\mathbb{E} u\left(x+X_{t}\right)=\int_{\mathbb{R}^{d}} u(x+y) p_{t}(y) d y, \quad t>0, x \in \mathbb{R}^{d} .
$$

If $u: \mathbb{R}^{d} \rightarrow \mathbb{R}$ is bounded and Borel measurable, then $P_{t} u$ is continuous, being convolution of a bounded function with an integrable function, cf. [21, Theorem 15.8]. In this section, we study the regularity of $x \mapsto P_{t} u(x)$ for unbounded functions $u$. If $u$ is unbounded, then we need some assumptions to make sense of the integral appearing in the definition of $P_{t} u$. It is natural to assume that there exists a constant $\beta>0$ such that the associated Lévy measure $v$ satisfies $\int_{|y| \geq 1}|y|^{\beta} v(d y)<\infty$. This condition ensures that $\mathbb{E}\left(\left|X_{t}\right|^{\beta}\right)<\infty$ for all $t \geq 0$, cf. Sato [20], and so $P_{t} u$ is well-defined for any function $u$ satisfying $|u(x)| \leq M\left(1+|x|^{\beta}\right), x \in \mathbb{R}^{d}$, for some $M>0$. Under the assumption that $p_{t} \in C_{b}^{1}\left(\mathbb{R}^{d}\right)$, we will show that $P_{t} u$ is locally Hölder continuous for every function $u$ satisfying $|u(x)| \leq M\left(1+|x|^{\gamma}\right), x \in \mathbb{R}^{d}$, for some $\gamma<\beta$. Before stating the result, let us give a word of caution. As

$$
P_{t} u(x)=\int_{\mathbb{R}^{d}} u(y) p_{t}(y-x) d y,
$$

a naive differentiation yields

$$
\nabla P_{t} u(x)=-\int_{\mathbb{R}^{d}} u(y) \nabla p_{t}(y-x) d y,
$$

and therefore one might suspect that $P_{t} u$ is differentiable (and not only locally Hölder continuous). In general, it is not possible to make this calculation rigorous, even if $u$ is 
bounded. To start with, it is not clear that the integral $\int_{\mathbb{R}^{d}}|u(y)|\left|\nabla p_{t}(y-x)\right| d y$ is finite since the decay of $p_{t}$ does not necessarily carry over to its derivatives. However, there is an interesting - and wide - class of Lévy processes for which the above reasoning can be made rigorous, and we will work out the details in the second part of this section.

Lemma 2 Let $\left(X_{t}\right)_{t \geq 0}$ be a Lévy process with Lévy triplet $(b, Q, v)$ and semigroup $\left(P_{t}\right)_{t \geq 0}$. Let $\beta>0$ be such that $\int_{|y| \geq 1}|y|^{\beta} v(d y)<\infty$, and assume that $X_{t}$ has for some $t>0$ a density $p_{t} \in C_{b}^{1}\left(\mathbb{R}^{\bar{d}}\right)$ with respect to Lebesgue measure. If $u$ is a measurable function satisfying $|u(x)| \leq M\left(1+|x|^{\gamma}\right)$ for some $M>0$ and $\gamma \in[0, \beta)$, then

$$
\left|P_{t} u(r x+r h)-P_{t} u(r x)\right| \leq C M r^{\gamma}|h|^{\varrho}, \quad|x|,|h| \leq 1, r \geq 1
$$

where $\varrho:=\frac{\beta-\gamma}{d+\beta} \in(0,1)$ and $C=C(t, \beta)<\infty$ is a constant which does not depend on u. In particular, $x \mapsto P_{t} u(x)$ is Hölder continuous of order $\varrho$ on any compact set $K \subseteq \mathbb{R}^{d}$ and

$$
\left\|P_{t} u\right\|_{C_{b}^{\varrho}(B(0, r))} \leq(C+2) M r^{\gamma} \quad \text { for all } r \geq 1
$$

Proof Because of the growth assumption on $u$, it follows from $\mathbb{E}\left(\left|X_{t}\right|^{\beta}\right)<\infty$ that $P_{t} u$ is well-defined. Fix $r, R \geq 1$ and $x, h \in \mathbb{R}^{d}$ with $|h|,|x| \leq 1$. By the definition of the semigroup,

$$
\begin{aligned}
\Delta_{h} & :=P_{t} u(r x+r h)-P_{t} u(r x) \\
& =\int_{\mathbb{R}^{d}} u(y)\left(p_{t}(y+r x+r h)-p_{t}(y+r x)\right) d y \\
& =r^{-d} \int_{\mathbb{R}^{d}} u(r z)\left(p_{t}(r z+r x+r h)-p_{t}(r z+r x)\right) d z .
\end{aligned}
$$

Thus, $\Delta_{h}=\Delta_{h}^{1}+\Delta_{h}^{2}$, where

$$
\begin{aligned}
& \Delta_{h}^{1}:=r^{-d} \int_{|z| \leq R} u(r z)\left(p_{t}(r z+r x+r h)-p_{t}(r z+r x)\right) d z, \\
& \Delta_{h}^{2}:=r^{-d} \int_{|z|>R} u(r z)\left(p_{t}(r z+r x+r h)-p_{t}(r z+r x)\right) d z .
\end{aligned}
$$

Applying the mean value theorem and using the growth condition on $u$, we find that

$$
\left|\Delta_{h}^{1}\right| \leq|h| r^{-d+1}\left\|\nabla p_{t}\right\|_{\infty} \int_{|z| \leq R}|u(r z)| d z \leq 2 M|h| r^{-d+1+\gamma}\left\|\nabla p_{t}\right\|_{\infty} R^{d+\gamma} .
$$

For the second term, we use again the growth condition on $u$ :

$$
\left|\Delta_{h}^{2}\right| \leq 4 M r^{-d+\gamma} \sup _{|h| \leq 1} \int_{|z|>R}|z|^{\gamma} p_{t}(r z+r x+r h) d z
$$




$$
\leq 4 M r^{-d+\gamma} R^{\gamma-\beta} \sup _{|h| \leq 1} \int_{\mathbb{R}^{d}}|z|^{\beta} p_{t}(r z+r h+r x) d z .
$$

Performing a change of variables and using the elementary estimate

$$
(a+b)^{\beta} \leq c_{\beta}\left(a^{\beta}+b^{\beta}\right), \quad a, b \geq 0,
$$

we get

$$
\begin{aligned}
\left|\Delta_{h}^{2}\right| & \leq 4 M r^{\gamma-\beta} R^{\gamma-\beta} \sup _{|h| \leq 1} \int_{\mathbb{R}^{d}}|y-(r x+r h)|^{\beta} p_{t}(y) d y \\
& \leq 42^{\beta} c_{\beta} M r^{\gamma} R^{\gamma-\beta}\left(1+\int_{\mathbb{R}^{d}}|y|^{\beta} p_{t}(y) d y\right) .
\end{aligned}
$$

Note that the integral on the right-hand side is finite since $\mathbb{E}\left(\left|X_{t}\right|^{\beta}\right)<\infty$. Consequently, we have shown that there exists a constant $C=C(\beta, t)>0$ such that

$$
\left|P_{t} u(r x+r h)-P_{t} u(r x)\right|=\left|\Delta_{h}\right| \leq C M r^{\gamma} R^{d+\gamma}|h|+C M r^{\gamma} R^{\gamma-\beta}
$$

for all $|h|,|x| \leq 1, r \geq 1$. Choosing $R:=|h|^{-1 /(d+\beta)}$ gives (11). The remaining assertion is obvious from (11).

If $\left(P_{t}\right)_{t \geq 0}$ is the semigroup associated with a subordinated Brownian motion $\left(X_{t}\right)_{t \geq 0}$, then the regularity estimate from Proposition 2 can be improved. We do not need this strengthened version for the proof of the Liouville theorem, but we present the proof since we believe that the result is of independent interest. Recall that a Lévy process $\left(S_{t}\right)_{t \geq 0}$ is a subordinator if $\left(S_{t}\right)_{t \geq 0}$ has non-decreasing sample paths.

Proposition 2 Let $\left(X_{t}\right)_{t \geq 0}$ be a Lévy process which is of the form $X_{t}=B_{S_{t}}$ for some d-dimensional Brownian motion $\left(B_{t}\right)_{t \geq 0}$ and a subordinator $\left(S_{t}\right)_{t \geq 0}$ satisfying $\mathbb{P}\left(S_{t}=0\right)=0$ for all $t>0$. Denote by $(b, Q, v)$ the Lévy triplet of $\left(X_{t}\right)_{t \geq 0}$, and let $\beta>0$ be such that $\int_{|y| \geq 1}|y|^{\beta} v(d y)<\infty$. If $u: \mathbb{R}^{d} \rightarrow \mathbb{R}$ is a measurable function satisfying $|u(x)| \leq M\left(1+|x|^{\gamma}\right), x \in \mathbb{R}^{d}$, for some $M>0$ and $\gamma \in[0, \beta]$, then $x \mapsto P_{t} u(x)$ is smooth for all $t>0$ and

$$
\left\|P_{t} u\right\|_{C_{b}^{k}(B(0, r))} \leq C_{k} M r^{\gamma} \quad \text { for all } r \geq 1, k \geq 1,
$$

where $C_{k}=C_{k}(t)$ is a finite constant, which does not depend on $u$ and $r$.

Let us mention that $\mathbb{P}\left(S_{t}=0\right)=0$ is equivalent to assuming that $\left(X_{t}\right)_{t \geq 0}$ has a density with respect to Lebesgue measure, cf. [14, Lemma 4.6].

Proof of Proposition 2 For $k \geq 1$ let $\left(B_{t}^{(k)}\right)_{t \geq 0}$ be a $k$-dimensional Brownian motion. The process $X_{t}^{(k)}:=B_{S_{t}}^{(k)}$ is a Lévy process with Lévy triplet, say, $\left(b^{(k)}, Q^{(k)}, v^{(k)}\right)$, cf. [22] or [20]. By definition, $X_{t}=X_{t}^{(d)}$ and $v=v^{(d)}$. Since $\left(B_{t}^{(k)}\right)_{t \geq 0}$ and $\left(S_{t}\right)_{t \geq 0}$ 
are independent, cf. [7, Theorem II.6.3], it follows from $B_{t}^{(k)}=\sqrt{t} B_{1}^{(k)}$ in distribution that

$$
\mathbb{E}\left(\left|B_{S_{t}}^{(k)}\right|^{\beta}\right)=\mathbb{E}\left(\left|S_{t}\right|^{\beta / 2}\right) \mathbb{E}\left(\left|B_{1}^{(k)}\right|^{\beta}\right) .
$$

Consequently,

$$
\int_{|y| \geq 1}|y|^{\beta} v^{(k)}(d y)<\infty \Longleftrightarrow \mathbb{E}\left(\left|B_{S_{t}}^{(k)}\right|^{\beta}\right)<\infty \Longleftrightarrow \mathbb{E}\left(\left|S_{t}\right|^{\beta / 2}\right)<\infty
$$

and so the finiteness of the fractional moment $\int_{|y| \geq 1}|y|^{\beta} v^{(k)}(d y)$ does not depend on the dimension $k$. By assumption, the moment is finite for $k=d$, and hence it is finite for all $k \geq 1$. Thus, $\mathbb{E}\left(\left|X_{t}^{(k)}\right|^{\beta}\right)<\infty$ for all $k \geq 1$ and $t \geq 0$. As $\mathbb{P}\left(S_{t}=0\right)$, the process $\left(X_{t}^{(k)}\right)_{t \geq 0}$ has a rotational invariant and smooth density $p_{t}^{(k)}(x)=p_{t}^{(k)}(|x|)$,

$$
\mathbb{P}\left(X_{t}^{(k)} \in d x\right)=p_{t}^{(k)}(|x|) d x
$$

and

$$
\frac{d}{d r} p_{t}^{(k)}(r)=-2 \pi p_{t}^{(k+2)}(r), \quad k \geq 1, r>0
$$

cf. [14, Corollary 3.2, Lemma 4.6]. Using polar coordinates, we get

$$
\int_{|x| \geq 1}|x|^{\gamma}\left|\nabla p_{t}^{(k)}(x)\right| d x=c \int_{r \geq 1} r^{\gamma+d} p_{t}^{(k+2)}(r) d r \leq c^{\prime} \mathbb{E}\left(\left|X_{t}^{(k+2)}\right|^{\gamma}\right)<\infty
$$

for all $\gamma \in[0, \beta]$ and $k \geq 1$. Since the continuous function $\left|\nabla p_{t}^{(k)}\right|$ is bounded on compact sets, this implies

$$
\int_{\mathbb{R}^{k}}\left(1+|x|^{\gamma}\right)\left|\nabla p_{t}^{(k)}(x)\right| d x<\infty \quad \text { for all } t \geq 0, \gamma \in[0, \beta], k \geq 1 .
$$

Applying iteratively (13) with $k=d+2 n, n \in \mathbb{N}$, we find that

$$
\int_{\mathbb{R}^{d}}\left(1+|x|^{\gamma}\right)\left|\partial^{\alpha} p_{t}^{(d)}(x)\right| d x<\infty
$$

for all $\gamma \in[0, \beta], t \geq 0$ and all multi-indices $\alpha \in \mathbb{N}_{0}^{d}$. Now we return to our original problem, i.e. we study the regularity of the semigroup $\left(P_{t}\right)_{t \geq 0}$ associated with $X_{t}=X_{t}^{(d)}$. Fix a measurable function $u$ with $|u(x)| \leq M\left(1+|x|^{\gamma}\right)$ for some constants $M>0$ and $\gamma \in[0, \beta]$. By definition,

$$
P_{t} u(x)=\mathbb{E} u\left(x+X_{t}\right)=\int u(y) p_{t}^{(d)}(y-x) d y, \quad x \in \mathbb{R}^{d} .
$$


By (14), we have $\int_{K} \int_{\mathbb{R}^{d}}|u(y)|\left|\partial_{x_{j}} p_{t}^{(d)}(y-x)\right| d y d x<\infty$ for every $j=1, \ldots, d$ and every compact set $K \subseteq \mathbb{R}^{d}$. Moreover, it follows by a similar reasoning to that the end of the proof of Lemma 1 that the mapping

$$
x \mapsto \int_{\mathbb{R}^{d}} u(y) \partial_{x_{j}} p_{t}^{(d)}(y-x) d y
$$

is continuous. Applying the differentiation lemma for parametrized integrals, cf. [15, Proposition A.1], we obtain that

$$
\partial_{x_{j}} P_{t} u(x)=-\int_{\mathbb{R}^{d}} u(y) \partial_{x_{j}} p_{t}^{(d)}(y-x) d y, \quad j=1, \ldots, d, x \in \mathbb{R}^{d}
$$

Performing a change of variables $y \rightsquigarrow y+x$, it is immediate from (14) and the growth condition on $u$ that $\left\|P_{t} u\right\|_{C_{b}^{1}(B(0, R))} \leq C M R^{\beta}, R \geq 1$, for some constant $C>0$. Iterating the procedure proves the assertion for higher order derivatives.

\section{Proof of Liouville's theorem}

In this section, we prove the Liouville theorem, cf. Theorem 1. First, we use a general result by Choquet and Deny [4] to show that the only bounded solutions to the convolution equation $P_{t} u=u$ are the trivial ones.

Proposition 3 Let $\left(X_{t}\right)_{t \geq 0}$ be a Lévy process with characteristic exponent $\psi$ and semigroup $\left(P_{t}\right)_{t \geq 0}$, and denote by $A f=-\psi(D) f$ the associated Lévy generator. Assume that $X_{t}$ has a density $p_{t} \in C_{b}\left(\mathbb{R}^{d}\right)$ for some $t>0$.

(i). If $u$ is a bounded measurable function such that $P_{t} u=u$ a.e., then $u$ is constant a.e.

(ii). (Liouville property) If $u \in L^{\infty}\left(\mathbb{R}^{d}\right)$ and $A u=0$ weakly, then $u$ is constant a.e.

Proof (i). Without loss of generality, we may assume that $P_{t} u(x)=u(x)$ for all $x \in \mathbb{R}^{d}$; otherwise replace $u$ by $\tilde{u}:=P_{t} u$ and note that $P_{t} u=P_{t} \tilde{u}$ as $X_{t}$ has a density with respect to Lebesgue measure. Since $\int_{\mathbb{R}^{d}} p_{t}(y) d y=1$ and $p_{t} \geq 0$ is continuous, there exist $x_{0} \in \mathbb{R}^{d}$ and $r>0$ such that $p_{t}(y)>0$ for all $y \in B\left(x_{0}, r\right)$. In particular, $B\left(x_{0}, r\right)$ is contained in the support of the distribution of $X_{t}$. By [4, Theorem 1], this implies

$$
u(x)=u(x+y) \quad \text { for all } x \in \mathbb{R}^{d}, y \in B\left(x_{0}, r\right)
$$

Hence, $u$ is constant.

(ii). This is immediate from Lemma 1 and (i).

We are now ready to prove the Liouville theorem. 
Proof of Theorem 1 By Lemma 1, we may assume without loss of generality that $u$ is continuous and $u(x)=P_{1} u(x)$ for all $x \in \mathbb{R}^{d}$. Applying Lemma 2, we find that there exists a constant $C>0$ such that

$$
\begin{aligned}
\left|u\left(r x^{\prime}+r h^{\prime}\right)-u\left(r x^{\prime}\right)\right| & =\left|P_{1} u\left(r x^{\prime}+r h^{\prime}\right)-P_{1} u\left(r x^{\prime}\right)\right|, \quad\left|h^{\prime}\right|,\left|x^{\prime}\right| \leq 1, r \geq 1 \\
& \leq C M r^{\gamma}\left|h^{\prime}\right|^{\varrho}
\end{aligned}
$$

for $\varrho:=(\beta-\gamma) /(d+\beta)>0$ and some constant $C=C(\beta)>0$. This implies

$$
|u(x+h)-u(x)| \leq 2 C M\left(1+|x|^{\gamma-\varrho}\right)|h|^{\varrho} \quad \text { for all } x \in \mathbb{R}^{d},|h| \leq 1 .
$$

Indeed: If $|x| \leq 1$, then this follows from (15) for $r=1, x^{\prime}=x$ and $h^{\prime}=h$; if $|x|>1$ we choose $r=|x|, h^{\prime}=h / r$ and $x^{\prime}=x / r$ in (15). This means that for each fixed $h \in \mathbb{R}^{d}, 0<|h| \leq 1$, the function $v(x):=|h|^{-\varrho}(u(x+h)-u(x))$ satisfies

$$
|v(x)| \leq 2 C M\left(1+|x|^{\gamma-\varrho}\right), \quad x \in \mathbb{R}^{d} .
$$

Since the semigroup $\left(P_{t}\right)_{t \geq 0}$ is invariant under translations, we have $P_{1} v=v$, and therefore we can apply the above reasoning to $v$ (instead of $u$ ) to obtain that

$$
|v(x+h)-v(x)| \leq 4 C^{2} M^{2}\left(1+|x|^{\gamma-2 \varrho}\right)|h|^{\varrho}, \quad x \in \mathbb{R}^{d},|h| \leq 1 .
$$

Define iteratively $\Delta_{h} u(x):=u(x+h)-u(x)$ and $\Delta_{h}^{k} u(x):=\Delta_{h}\left(\Delta_{h}^{k-1} u\right)(x), k \geq 2$, then the previous inequality shows

$$
\left|\Delta_{h}^{2} u(x)\right| \leq 4 C^{2} M^{2}\left(1+|x|^{\gamma-2 \varrho}\right)|h|^{2 \varrho}, \quad x \in \mathbb{R}^{d},|h| \leq 1 .
$$

Iterating the procedure, we find that

$$
\left|\Delta_{h}^{k} u(x)\right| \leq(2 C M)^{k}\left(1+|x|^{\gamma-k \varrho}\right)|h|^{k \varrho}, \quad x \in \mathbb{R}^{d},|h| \leq 1,
$$

for the largest integer $k \geq 1$ such that $\gamma-k \varrho \geq 0$; the latter condition ensures that the constant $\gamma$ in Lemma 2 is non-negative. Applying once more Lemma 2, we get

$$
\left|\Delta_{h}^{k} u\left(r x^{\prime}+r h^{\prime}\right)-\Delta_{h}^{k} u\left(r x^{\prime}\right)\right| \leq(2 C M)^{k+1} r^{\gamma-k \varrho}\left|h^{\prime}\right|^{\varrho}|h|^{k \varrho}, \quad\left|x^{\prime}\right|,\left|h^{\prime}\right| \leq 1, r \geq 1 .
$$

If $x, h \in \mathbb{R}^{d}$ are such that $|x| \geq 1$ and $|h| \leq 1$, then we obtain from this inequality for $r=|x|, x^{\prime}=x / r$ and $h^{\prime}=h / r$ that

$$
\left|\Delta_{h}^{k} u(x+h)-\Delta_{h}^{k} u(x)\right| \leq(2 C M)^{k+1}|x|^{\gamma-(k+1) \varrho}|h|^{(k+1) \varrho} .
$$

As $\gamma-(k+1) \varrho<0$, this gives

$$
\sup _{|x|>r}\left|\Delta_{h}^{k+1} u(x)\right| \leq(2 C M)^{k+1} r^{\gamma-(k+1) \varrho}|h|^{(k+1) \varrho \stackrel{r \rightarrow \infty}{\longrightarrow}} 0 .
$$


Consequently, $x \mapsto w(x):=\Delta_{h}^{k+1} u(x)$ is for each fixed $|h| \leq 1$ a continuous function which vanishes at infinity and which satisfies $P_{1} w=w$. The Liouville property, cf. Proposition 3, yields $w=0$, i.e. $\Delta_{h}^{k+1} u(x)=0$ for all $x \in \mathbb{R}^{d}$ and $|h| \leq 1$. We claim that this implies that $u$ is a polynomial. Take $\varphi \in C_{c}^{\infty}\left(\mathbb{R}^{d}\right)$ with $\varphi \geq 0$ and $\int_{\mathbb{R}^{d}} \varphi(x) d x=1$, and set $\varphi_{n}(x):=n^{d} \varphi(n x)$. The convolution $u_{n}:=u * \varphi_{n}$ satisfies $\Delta_{h}^{k+1} u_{n}(x)=0$ for all $x \in \mathbb{R}^{d}$ and $|h| \leq 1$. Since $u_{n}$ is smooth, we have

$$
\partial_{x_{j}}^{k+1} u_{n}(x)=\lim _{r \downarrow 0} \frac{\Delta_{r e_{j}}^{k+1} u_{n}(x)}{r^{k+1}}=0
$$

for all $x \in \mathbb{R}^{d}, j \in\{1, \ldots, d\}$ and $n \in \mathbb{N}$; here $e_{j}$ denotes the $j$-th vector in $\mathbb{R}^{d}$. Hence, $\partial^{\alpha} u_{n}=0$ for all $|\alpha| \geq N:=(k+1) d$, and so $u_{n}$ is a polynomial of degree at most $N$ for each $n \in \mathbb{N}$. Since $u_{n}$ converges pointwise to $u$, it follows that $u$ is a polynomial of degree at most $N$. Recalling that $u$ satisfies by assumption the growth condition $|u(x)| \leq M\left(1+|x|^{\gamma}\right)$ for all $x \in \mathbb{R}^{d}$, we conclude that $u$ is a polynomial of order at most $\lfloor\gamma\rfloor$.

Acknowledgements I am grateful to Prof. Niels Jacob and an anonymous referee for comments which helped to improve the presentation of this paper.

Funding Open Access funding enabled and organized by Projekt DEAL.

Open Access This article is licensed under a Creative Commons Attribution 4.0 International License, which permits use, sharing, adaptation, distribution and reproduction in any medium or format, as long as you give appropriate credit to the original author(s) and the source, provide a link to the Creative Commons licence, and indicate if changes were made. The images or other third party material in this article are included in the article's Creative Commons licence, unless indicated otherwise in a credit line to the material. If material is not included in the article's Creative Commons licence and your intended use is not permitted by statutory regulation or exceeds the permitted use, you will need to obtain permission directly from the copyright holder. To view a copy of this licence, visit http://creativecommons.org/licenses/by/4.0/.

\section{References}

1. Alibaud, N., del Teso, F., Endal, J., Jakobsen, E.R.: The Liouville theorem and linear operators satisfying the maximum principle. J. Math. Pures Appl. 142, 229-242 (2020)

2. Barlow, M.T., Bass, R.F., Gui, C.: The Liouville property and a conjecture of De Georgi. Commun. Pure Appl. Math. 53, 1007-1038 (2000)

3. Cheng, T., Chen, W., Li, C., Zhang, L.: A Liouville theorem for $\alpha$-harmonic functions in $\mathbb{R}_{+}^{n}$. Discrete Contin. Dyn. Syst. 36, 1721-1736 (2015)

4. Choquet, G., Deny, J.: Sur léquation de convolution $\mu=\mu * \sigma$. C. R. Acad. Sci. Paris 250, 799-801 (1960)

5. Fall, M.M., Weth, T.: Liouville theorems for a general class of nonlocal operators. Potential Anal. 45, 187-200 (2016)

6. Hulanicki, A.: A class of convolution semi-groups of measures on a Lie group. In: Weron, A. (ed.) Probability Theory on Vector Spaces, pp. 82-101. Springer, Berlin (1980)

7. Ikeda, N., Watanabe, S.: Stochastic Differential Equations and Diffusion Processes. North-Holland, Amsterdam (1992)

8. Jacob, N.: Pseudo Differential Operators and Markov processes I: Fourier Analysis and Semigroups. World Scientific, Singapore (2001)

9. Khoshnevisan, D., Schilling, R.L.: From Lévy-Type Processes to Parabolic SPDEs. Birkhäuser, Basel (2016) 
10. Knopova, V., Schilling, R.L.: A note on the existence of transition probability densities of Lévy processes. Forum Math. 25, 125-149 (2013)

11. Knopova, V., Schilling, R.: On the Liouville property for non-local Lévy generators. arXiv:1909.01237

12. Kühn, F.: Existence and estimates of moments for Lévy-type processes. Stoch. Proc. Appl. 127, 10181041 (2017)

13. Kühn, F.: Schauder estimates for equations associated with Lévy generators. Integr. Equ. Oper. Theory 91, 10 (2019)

14. Kühn, F., Schilling, R.L.: A probabilistic proof of Schoenberg's theorem. J. Math. Anal. Appl. 476, 13-26 (2019)

15. Kühn, F., Schilling, R.L.: Strong convergence of the EulerMaruyama approximation for a class of Lévy-driven SDEs. Stoch. Proc. Appl. 129, 2654-2680 (2019)

16. Liang, M., Wang, J.: Spatial regularity of semigroups generated by Lévy type operators. Math. Nachr. 292, 1551-1566 (2019)

17. Masamune, J., Uemura, T.: $L^{p}$-Liouville property for non-local operators. Math. Nachr. 284, 22492267 (2011)

18. Priola, E., Zabczyk, J.: Liouville theorems for non-local operators. J. Funct. Anal. 216, 455-490 (2004)

19. Ros-Oton, X., Serra, J.: Regularity theory for general stable operators. J. Differ. Equ. 260, 8675-8715 (2016)

20. Sato, K.: Lévy Processes and Infinitely Divisible Distributions. Cambridge University Press, Cambridge (2013). (revised ed)

21. Schilling, R.L.: Measures, Integrals and Martingales, 2nd edn. Cambridge University Press, Cambridge (2017)

22. Schilling, R.L., Song, R., Vondraček, Z.: Bernstein Functions: Theory and Applications, 2nd edn. De Gruyter, Berlin (2012)

23. Schilling, R.L., Wang, J.: On the coupling property and the Liouville theorem for OrnsteinUhlenbeck processes. J. Evol. Equ. 12, 119-140 (2012)

Publisher's Note Springer Nature remains neutral with regard to jurisdictional claims in published maps and institutional affiliations. 